\title{
WIDE FIELD STAR COUNT MAPPING OF LARGE SCALE AREAS OF STAR FORMATION IN THE LARGE MAGELLANIC CLOUD
}

\author{
E. KONTIZAS ${ }^{1}$, S.E. MARAVELIAS ${ }^{1,2}$, A. DAPERGOLAS ${ }^{1}$, \\ Y. BELLAS-VELIDIS ${ }^{1}$ and M. KONTIZAS ${ }^{2}$ \\ ${ }^{1}$ Astronomical Institute, National Observatory of Athens, P. O. Box 20048, \\ GR - 118 10, Thesseion, Athens, Greece \\ 2 Section of Astrophysics, Astronomy and Mechanics, Department of Physics, \\ University of Athens, Panepistemiopolis, GR - 157 84, Zographos, Athens, Greece
}

Star formation in galaxies is a major astrophysical problem which can be investigated in several ways. The distribution and loci of all kinds of young objects, including OB associations, young clusters, HII regions, GMCs, Bok globules, dark clouds, dust lanes, protostars, as well as YSOs detected in NIR and FIR surveys constitute the principal signposts for this investigation. The individual nature of all these objects has been and is still continously studied. However it is also extremely interesting to associate the coexistence of these objects, and their relation to the structure of the parent galaxy. Such studies have been carried out by several investigators and are frequently summarized when star formation processes are examined.

Considering the morphology of a galaxy and its main components (i.e. spiral arms, central bulge and/or bar), several investigations are focused on whether the young objects form large structures, which are directly related to the main morphological type of the parent galaxy. Ambartsumyan et al. (1963) and Shakhbazyan (1968) have used the term 'superassociation' for groups of genuine associations which were members of larger structures and later on Efremov (1988) introduced the concept of 'stellar complexes' and 'supercomplexes', these being gigantic structures containing the youngest population objects in a galaxy.

In this project, an investigation has started concerning the LMC stellar complexes using wide field photographic plates taken with the $1.2 \mathrm{~m}$ U.K. Schmidt Telescope. Two approaches have been used in order to study the existence of such structures and define their boundaries: (i) star counts in various wavelength ranges to reveal the excess of star number density in several large areas of the LMC, down to a limiting magnitude of $\approx 21^{\mathrm{m}} .0$; and (ii) spectral classification of stars in the same areas, down to magnitude $B \approx 18$. 5 . Iso-contours and the distribution of $O B$ stars in those fields have revealed the existence of such superstructures, where there is a higher density of stars and a significantly larger ratio of OB stars to RSGs, than in the surrounding fields. Smaller structures, with a typical dimension of $\approx 0.2 \mathrm{kpc}$, coinciding with the 'aggregates' of Efremov (Maravelias et al. 1991, 1992), seem also to exist there. In this investigation an area of $\approx 1.8 \mathrm{kpc} \times 1.2 \mathrm{kpc}$ around Shapley $\mathrm{IX}$ has been studied. This is a very extended structure, relatively rich in $\mathrm{OB}$ associations, gas, and young clusters. It has been deduced from the detailed study of this region that the iso-contours (which are not shown here), taken from star counts on a $U$ plate exhibit the following characteristics: (i) the existence of an extended region, dense in number of stars, which exceeds the mean density of the surrounding 
field by $2 \sigma$; (ii) a high proportion of OB stars; and (iii) a clear deficiency of RSGs, which is illustrated in Fig. 1, where the distribution of spectral types is given for the area of the giant complex (i.e. the area inside the minimum contour, taken as $13 * / 120 \mathrm{pc}^{2}$ ), and that of the surrounding field, normalized for the same area and corrected for completeness. Ivanov (1991) has reported similar properties for complexes and supercomplexes in M33.

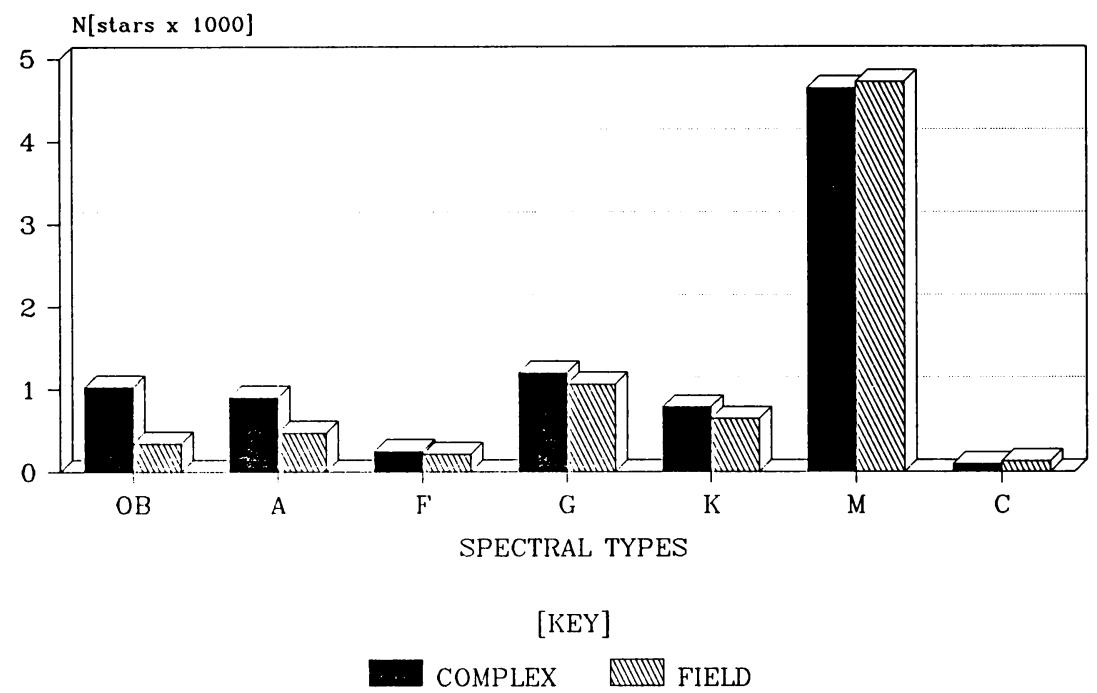

Figure 1. Distribution of spectral types in Shapley IX.

\section{References}

Ambartsumyan, V.A., Iskudayan, S.G., Shakhbazyan, R.K. and Sahakyan, K.A., 1963. Bull. Byurakan Obs., 33, 1.

Efremov, Yu. N., 1988. Sov. Sci. Rev. E. Astrophys. Space Phys., $7^{2}, 105$.

Ivanov, G.R., 1991. A \& SS, 178, 227.

Maravelias, S.E., Kontizas, M., Kontizas, E. and Dapergolas, A., 1991. In 'Proceedings of the 1st General Conference of the Balkan Physical Union', ed. K.M.Paraskevopoulos, 1, 460, (Hellenic Physical Society - Thessaloni Branch).

Maravelias, S.E., Kontizas, M., Kontizas, E. and Dapergolas, A., 1992. In 'The Feedback of Chemical Evolution on the Stellar Content of Galaxies', eds. D. Alloin and G. Stasinska, p. 254 (Observatoire de Paris).

Shakhbazyan, R.K., 1968. Astrofizika, 42, 273. 